\title{
Validating and determining the weight of items used for evaluating clinical governance implementation based on analytic hierarchy process model
}

\author{
Elaheh Hooshmand ${ }^{1}$, Sogand Tourani ${ }^{2,3}$, Hamid Ravaghi ${ }^{3}$, Ali Vafaee Najar ${ }^{1}$, Marziye Meraji ${ }^{4}$, \\ Hossein Ebrahimipour ${ }^{*}$
}

\section{Abstract}

Background: The purpose of implementing a system such as Clinical Governance (CG) is to integrate, establish and globalize distinct policies in order to improve quality through increasing professional knowledge and the accountability of healthcare professional toward providing clinical excellence. Since CG is related to change, and change requires money and time, CG implementation has to be focused on priority areas that are in more dire need of change. The purpose of the present study was to validate and determine the significance of items used for evaluating CG implementation.

Methods: The present study was descriptive-quantitative in method and design. Items used for evaluating CG implementation were first validated by the Delphi method and then compared with one another and ranked based on the Analytical Hierarchy Process (AHP) model.

Results: The items that were validated for evaluating CG implementation in Iran include performance evaluation, training and development, personnel motivation, clinical audit, clinical effectiveness, risk management, resource allocation, policies and strategies, external audit, information system management, research and development, CG structure, implementation prerequisites, the management of patients' non-medical needs, complaints and patients' participation in the treatment process. The most important items based on their degree of significance were training and development, performance evaluation, and risk management. The least important items included the management of patients' non-medical needs, patients' participation in the treatment process and research and development.

Conclusion: The fundamental requirements of CG implementation included having an effective policy at national level, avoiding perfectionism, using the expertise and potentials of the entire country and the coordination of this model with other models of quality improvement such as accreditation and patient safety.

Keywords: Validating, Evaluation, Implementation, Clinical Governance (CG), Analytic Hierarchy Process (AHP)

Copyright: (C) 2015 by Kerman University of Medical Sciences

Citation: Hooshmand E, Tourani S, Ravaghi H, Vafaee Najar A, Meraji M, Ebrahimipour H. Validating and determining the weight of items used for evaluating clinical governance implementation based on analytic hierarchy process model. Int J Health Policy Manag. 2015;4(10):645-651. doi:10.15171/ijhpm.2015.79

\section{Article History:}

Received: 9 October 2014

Accepted: 4 April 2015

ePublished: 8 April 2015

\section{Key Messages}

Implications for policy makers

- Several key obstacles stand in the way of Clinical Governance (CG) implementation in Iran: a regressive financing system; divisions inside the involved institution; absence of a standard definition for benefit packages; managerial deficiencies and time limitations.

- To prioritize areas of CG that are in the greatest need of change through the Analytic Hierarchy Process (AHP) model and the Delphi method.

Implications for public

To identify items that are essential in evaluating the Clinical Governance (CG) system and help its effective implementation in the healthcare system of Iran. It is expected that with proper implementation, in accordance with the conditions of healthcare system in Iran, CG system be able to solve some of the healthcare problems.

\section{Background}

The phrase "Clinical Governance" (CG) was first introduced by the National Health Service (NHS) in the UK and soon gained international acceptance as a general framework for improving quality through increasing professional knowledge and the accountability of healthcare professionals toward providing clinical excellence $(1,2)$. The necessity of adopting CG as a qualitative approach to providing healthcare was determined by research in response to the lack of strict standards and the quality issues with the existing system $(1,3,4)$ and the urgent need for improving the quality of care (5-10). In brief, healthcare organizations are required 
to improve the quality of care through combining activities like performance control and ensuring clinical quality at all organizational levels (11). This framework increases managers' responsibility and accountability toward the leading and coordination of implementation strategies for improving the quality of care (12).

The purpose of implementing a system such as CG is to generally integrate, establish and globalize distinct policies in order to develop an organization in which the final responsibility and accountability toward CG lies with the executive managers and is considered part of the daily responsibilities of healthcare staff (13). All healthcare organizations should implement this structure and ensure the establishment of effective communication around it (14). CG also includes older approaches to improving the quality of care, such as performance audit, use of clinical guidelines, risk management and professional development; however, it involves more than them alone (15).

After examining recent studies conducted in Iran on this subject and the efforts made to date, the health policy council of the Ministry of Health and Medical Education (MoHME) developed the CG program on October 29th, 2009, comprising of seven main components (16). The main challenge in the implementation of CG is to convert policies into implementable practices (16). Designing a system for the evaluation of CG implementation that helps ensure the successful building of the infrastructure and settings required for these goals is an essential matter (17).

Different countries have used different models for evaluating their CG systems; these models assess the rate of healthcare organizations' success in implementing CG (18). Models proposed by Grainger et al. and Specchia et al. and some Australian and UK models are worth noting (18-21). A study conducted in Iran in 2013 by Hooshmand et al. identified the key items for a successful evaluation of CG implementation based on the views of CG experts (22). The results of this study showed that assessing CG implementation has different aspects and is influenced by many factors. Since CG is related to change, and change requires money and time, its implementation must be focused on priority areas that are in dire need for the greatest changes (22). In recent years, the MoHME has developed standards for the evaluation of CG implementation; however, it has not set a certain order of priority for them. As a result, the present study was conducted with the purpose of validating and prioritizing the items used for evaluating CG implementation based on the Analytical Hierarchy Process (AHP) model and investigating the existence of a relationship between the MoHME defined standards and the standards that have obtained the highest AHP scores; in other words, assessing whether the MoHME standards obtained the highest AHP scores too or not. The results of the present study could help identify items that are essential in the evaluation of CG and its effective implementation in the Iranian healthcare system.

\section{Methods}

The present study was descriptive-quantitative in design. Items effective for evaluating CG implementation were first validated by the Delphi method and then compared with one another and ranked based on the AHP model.
For the Delphi stage of the study, 30 individuals with similar educational backgrounds with at least 6 months of work experience in the CG evaluation committee were selected as subjects. As Delphi panelists need to be concerned experts in the field and as the results of Delphi studies cannot be generalized, the use of a select list of panelists is unlikely to bias the results and is considered a good first step to selecting both committed and knowledgeable panel members. For these reasons, the researchers asked a well-recognized expert (the head of the CG committee of the MoHME) to provide a list of potential panelists, which could possibly have a number of benefits.

First, a recommended individual with enough experience and credibility to recognize and choose other qualified panelists for the Delphi stage helped identify experts or colleagues specialized in the field. Second, as a reference for the project, he assisted the researchers in contacting the target panelists with an initial introduction to both the researcher and the research subject. Before the Delphi researchers contacted the potential panelists, the recommended individual directly contacted the qualified experts over the telephone or by email, which helped significantly reduce the researchers' chance of being declined or receiving no response by the prospective candidates for their call inviting for participation. In the AHP stage, participants included 20 CG managers at teaching hospitals and members of the central CG committee of Mashhad University of Medical Sciences (MUMS). These participants, too, were selected based on the similarity of their educational backgrounds, their research area of focus and their work experience. Considering the time limitations and the lack of access to every single national expert in this field, the AHP stage was carried out with the sole participation of MUMS' CG authorities. MUMS is one of the top universities of Iran with a positive performance at the CG Fair and with CG authorities who have completed their training courses outside Iran and who are considered experts in the field. This study was conducted using two researcher-designed questionnaires with their validity evaluated by the content validity method and their reliability by Cronbach alpha ${ }^{1}$, which is shown in result section (23).

The item validating questionnaire used for the evaluation of Clinical Governance (CG) implementation based on the Delphi model

This questionnaire was designed based on the items used for the evaluation of CG implementation in Iran (22). Table 1 show the items used in the Delphi stage. The Delphi method is a systematic forecasting method that involves structured interaction among a group of experts on a subject. The Delphi technique typically includes at least two rounds of experts answering questions and giving justification for their answers, providing the opportunity between rounds for changes and revisions. The multiple rounds, which are stopped after a pre-defined criterion is reached, enable the group of experts to arrive at a consensus forecast on the subject being discussed (24).

The scoring system includes scores ranging from 0 to 100 . After the data analysis, items that obtained scores below $60 \%$ of the total score were revised according to the expert comments (25). The revised items were offered for a re- 
Table 1. Delphi components

\begin{tabular}{l}
\hline Category \\
\hline Human resources management \\
Performance evaluation \\
Training and development \\
Personnel motivation \\
Improving clinical quality \\
Clinical audit \\
Clinical effectiveness \\
Risk management \\
Development management \\
Allocation of resources \\
Policies and strategies \\
External audit \\
Information systems \\
Research activities \\
Establishing CG \\
CG structure \\
CG prerequisites \\
Providing patient-oriented healthcare \\
Management of patients' non-medical needs \\
\hline CG= Clinical Governance. \\
\hline
\end{tabular}

$\mathrm{CG}=$ Clinical Governance.

survey. The researcher continued performing this step until he/she attained a consensus of opinion. To collect data for the Delphi stage, the questionnaire was offered to participants in person except in the case of 5 experts who received it by email. An official letter introducing the researcher and the study objectives and inviting participants to fill out the questionnaire was attached, as well. After the re-survey, the mean score of participants' opinion for each item reached $85 \%$ and their standard deviation was calculated to be $20 \%$; therefore, agreement in opinion was reached and the Delphi stage was successfully completed in two steps.

The evaluation questionnaire based on the pair wise comparisons using the Analytical Hierarchy Process (AHP) model

AHP model was used to determine the significance of each item identified as essential in the implementation of CG. Each significant factor in CG implementation identified through the pair wise comparisons and according to the experts' opinions was compared and ranked according to the AHP model in Expert Choice software. To perform the pair wise comparison and carry out the model's steps, factors and variables were presented from more general to more specific in a paired comparison matrix and were then evaluated in groups by the experts.

In the paired comparison, the respondent compared the factors inserted in each row with the factors inserted in each column, and if these two were equally significant for or exerted an equal influence on the general factor on top of the table, number 1 was inserted in the intersection cell of the column and the row (i.e. the two factors). If the row factor was more significant or exerted a greater influence than the column factor, a number between 2 to 9 was placed in the intersection cell. However if the column factor was more significant or exerted a greater influence than the row factor, the fraction $1 / 9$ was inserted in the intersection cell. The insertion of fractions shows that the row factor is less significant or exerts less influence than the column factor. To collect data for the pair wise comparison, a two-hour session was held in which instructions for filling out the questionnaire were first provided and then participants were asked to enter their opinion about the significance of each criterion in the questionnaire.

In the Delphi stage, data were analyzed in SPSS 16 software using descriptive statistics (the mean of the scores and the standard deviation), and in the pair wise comparison stage, data were analyzed in Expert Choice software.

Results

Table 2 shows the general characteristics of participants of the Delphi stage of the study.

Of the total of 30 participants, 3 were General Practitioners (GPs), 5 had bachelor's degree, 14 had master's degrees and 6 had $\mathrm{PhD}$ degrees.

Table 3 shows the working experience and the age group of participants of the Delphi stage.

As shown in Table 2 and 3, participants had more than 3 years of working experience in CG, 23 had 1 to 3 years, 4 had less than 1 year and the mean age of participants was 33.42.

In the AHP stage, all CG authorities of teaching hospitals (a total of 11 participants) and members of the central CG committee of MUMS (a total of 9 participants) were selected through the purposive method and based on the determined inclusion criteria.

Table 4 shows the demographic characteristics of participants of the AHP stage of the study. As shown by this table, of the total of 20 participants, 3 were GPs responsible for the implementation of CG, 5 of those with master's degrees were CG experts, and 12 of those with bachelor's degrees were nurses. The mean age of participants at this stage was 45.26 . Table 5 shows the result of Cronbach alpha. The theoretical value of alpha varies from 0 to 1 , since it is the ratio of two

Table 2. Demographic characteristics of participants of the Delphi stage

\begin{tabular}{lccccc}
\hline $\begin{array}{l}\text { Level of } \\
\text { education }\end{array}$ & GP & $\begin{array}{c}\text { Bachelor's } \\
\text { degree }\end{array}$ & $\begin{array}{c}\text { Master's } \\
\text { degree }\end{array}$ & $\begin{array}{c}\text { PhD } \\
\text { degree }\end{array}$ & \multirow{2}{*}{ Total } \\
\cline { 2 - 5 } & No. (\%) & No. (\%) & No. (\%) & No. (\%) & \\
\hline Gender & & & & & \\
$\quad$ Female & $0(0.00)$ & $2(6.68)$ & $10(33.33)$ & $2(6.61)$ & 14 \\
$\quad$ Male & $3(10.00)$ & $3(10.00)$ & $4(6.61)$ & $6(13.30)$ & 16 \\
Total & $3(10.00)$ & $5(16.74)$ & $16(53.30)$ & $6(20.00)$ & 30 \\
\hline
\end{tabular}

$\mathrm{GP}=$ General practitioner.

Table 3. Working experience and age group of participants of the Delphi stage

\begin{tabular}{lcccc}
\hline Working & Less than 1 year & $\mathbf{1}$ to 3 years & Up to 3 years & \multirow{2}{*}{ Total } \\
\cline { 2 - 4 } experience & No. (\%) & No. (\%) & No. (\%) & \\
\hline Age & & & & \\
$20-30$ & $3(27.24)$ & $8(72.71)$ & $0(0.00)$ & 11 \\
$31-40$ & $1(9.00)$ & $9(81.80)$ & $1(9.00)$ & 11 \\
$41-50$ & $0(0.00)$ & $6(75.00)$ & $2(25.00)$ & 8 \\
Total & $4(13.33)$ & $23(76.66)$ & $3(10.00)$ & 30 \\
\hline
\end{tabular}


Table 4. Demographic characteristics of participants of the AHP stage

\begin{tabular}{lllll}
\hline \multirow{2}{*}{$\begin{array}{l}\text { Education } \\
\text { level }\end{array}$} & Bachelor degree & Master degree & GP & \multirow{2}{*}{ Total } \\
\cline { 2 - 4 } & No. (\%) & No. (\%) & No. (\%) & \\
\hline Gender & & & & \\
Female & $11(57.88)$ & $5(26.30)$ & $2(10.53)$ & 18 \\
Male & $1(5.26)$ & $0(0.00)$ & $1(5.26)$ & 2 \\
Total & $12(63.15)$ & $5(26.30)$ & $3(15.97)$ & 20 \\
\hline
\end{tabular}

$\mathrm{GP}=$ General practitioner; AHP= Analytical Hierarchy Process.

Table 5. Results of Cronbach alpha

\begin{tabular}{llcc}
\hline No. & Category & Sub-category & Cronbach alpha \\
\hline 1 & Human resources management & 3 & 82.20 \\
2 & Improving clinical quality & 3 & 70.10 \\
3 & Developing management & 5 & 72.32 \\
4 & Establishing CG & 2 & 73.41 \\
5 & Providing patient-oriented & 3 & 76.16 \\
\hline
\end{tabular}

$\mathrm{CG}=$ Clinical Governance.

variances. However, depending on the estimation procedure used, estimates of alpha can take on any value less than or equal to 1 , including negative values, although only positive values make sense (23). Higher values of alpha are more desirable. Some professionals, as a rule of thumb, require a reliability of 0.70 or higher (obtained on a substantial sample) before they will use an instrument (23). Table 5 shows the results of Cronbach alpha in different dimensions, which are all acceptable.

The accredited criteria for evaluating CG implementation in Iran included performance evaluation, training and development, personnel motivation, clinical audit, clinical effectiveness, risk management, resource allocation, policies and strategies, external audit, information systems, research activities, CG structures, prerequisites of CG implementation, managing patients' non-medical needs, complaints and patients' participation in the treatment process.

Table 6 shows the significance of each criteria based on the experts' points of view. Findings of this stage of the study show that, from the experts' points of view, the most significant criteria were training and development, performance evaluation and risk management, and the least significance was given to patient participation, managing patients' non-medical needs and research and development. The inconsistency rate was 0.07 in this comparison, which falls in the accepted range.

\section{Discussion}

The present descriptive-quantitative study aimed to validate and determine the significance of factors used for evaluating CG implementation based on the AHP model. This section discusses the results of the study based on the main categories in CG evaluation and the criteria that obtained the highest scores in each of the domains and the measures taken by the MoHME with regard to each criterion.

Main category 1: human resources management

In this category, the highest scores pertain to the training and development and the performance evaluation criteria.
Table 6. The determined significance of each of the criteria used for evaluating CG implementation from the experts' points of view

\begin{tabular}{ll}
\hline Sub-concept & $\boldsymbol{P}$-value \\
\hline Performance evaluation & 0.12 \\
Training and development & 0.64 \\
Personnel motivation & 0.09 \\
Clinical audit & 0.05 \\
\hline Clinical effectiveness & 0.05 \\
Risk management & 0.10 \\
Resource allocation & 0.07 \\
Policies and strategies & 0.03 \\
Information systems & 0.05 \\
Research activities & 0.02 \\
CG structures & 0.06 \\
Prerequisites of CG implementation & 0.05 \\
\hline Managing patients' non-medical needs & 0.02 \\
Complaints & 0.03 \\
Patients' participation in the treatment process & 0.02 \\
\hline CG= Clinical Governance. &
\end{tabular}

As the first step in CG implementation is arranging training courses (15), the Iranian MoHME also stressed the significance of this criterion and put even more emphasis on developing personnel development plans for revising educational programs (26).

Furthermore, the results of a study conducted by Specchia et al. showed that performance evaluation is of great importance in all healthcare organizations $(19,27)$.

In general, the results of the study indicate that the criteria pertaining to Human resources management (category 1) have obtained the highest scores among all the other criteria assessed during the AHP process.

\section{Main category 2: improving clinical quality}

Risk management and patient safety comprise one of the main priorities in this category, especially given that the concept of risk management in CG has been emphasized at a national level as the backbone of the health industry.

Clinical effectiveness is ranked next in significance according to the AHP results. Clinical effectiveness is in fact one of the essential components of CG for improving and guaranteeing quality and is related to choosing the right action at the right time and for the right patient and is associated with improved quality and performance $(28,29)$.

The other criterion in this category is clinical audit, which, according to AHP experts, is equal in significance to clinical effectiveness. For this very reason, every study conducted on this topic has considered clinical audit as one of the main components of risk management and clinical effectiveness $(17,29)$. This criterion has been well-addressed by the MoHME under the clinical audit group.

\section{Main category 3: development management}

The highest scores in this category pertained to resource allocation. The significance of this criterion lies in how CG can change performances only if additional resources are allocated to this purpose or if the existing resources are re-allocated $(30,31)$. This criterion is not addressed in the developed program of the MoHME, since, in Iran, CG implementation is 
the responsibility of the Medical Care Deputy, while resource allocation is performed by the Planning Deputy; which is precisely why having universal governance is an integral component of an effective CG implementation.

Next in significance from this category are information systems. The complexity of problems that healthcare is faced with today has increased the need for acquiring relevant information for decision-making more than any other time (18). In the criteria developed by the MoHME for CG evaluation, this criterion was placed under the group of information use, and the necessity of having a Hospital Information System (HIS) was more emphasized.

Policies and strategies comprise the next priority in this domain. Developing policies and strategies shapes the framework for achieving goals and attaining the results expected of CG implementation (32). In the developed program of the MoHME, this criterion was placed under the management group and given the name "developing strategic plans" and addressed strategic, practical planning for hospitals with an emphasis on CG.

\section{Main category 4: establishing Clinical Governance (CG)}

This domain introduces two main criteria of almost equal significance according to experts. The first one is CG structure, which guarantees system accountability (33). This criterion was developed by the MoHME under the management group and addressed the presence of CG groups and offices in hospitals. The second one is CG prerequisites, which ensure managers' commitment to CG (34). These two criteria show that the required infrastructures for the establishment of CG are developed at given organizations.

\section{Main category 5: patient-oriented health services}

This category addresses complaints, the management of patients' non-medical needs and patients' participation in the treatment process, with the last two being of equal significance according to experts. Complaint management was placed by the MoHME under the interaction with the patients and the community group and given the name "complaint investigation". Relevant studies have emphasized the importance of active follow-up of complaints and displaying the results of complaint investigations at hospitals (35-38). Performing external audits and using their results for improving the quality of care is another criterion obtained by the AHP results for the evaluation of CG implementation. Nayar and Ozcan believed that senior managers and executive teams of healthcare organizations have to showcase the standard implementation of the CG system in their organizations through external audits (38).

The next criterion in this domain is patients' participation. A number of studies have also emphasized the essential role of patients' participation in the treatment process, the necessity of patient training and providing proper information to patients for encouraging their participation in the therapeutic process for evaluating CG implementation $(1,17,39,40)$. The MoHME has also confirmed these necessities.

Management of patients' non-medical needs is the next criterion that was addressed in the developed program of the MoHME and was integrated with the previous criterion and given the name "welcoming patients and filling informed consent forms". Studies conducted on this subject have shown that these criteria can guarantee more modern and patient-centered health services along with clinical excellence $(7,41-43)$.

\section{Study limitations}

The present study was conducted with the qualitative approach and with a limited number of samples selected through the purposive method. Although samples were chosen from MUMS, which is a top university with a positive performance at the CG Fair and with CG authorities who have completed their training courses outside of Iran and are experts of the field, the results of the study cannot be generalized to the entire country.

At the Delphi stage, the definition of certain conditions and criteria for selecting experts might have led to the exclusion of a number of experts and the study has inevitably missed out on their comments and opinions.

In the AHP stage of the study, relative value depends on the options and the exerting of preferences. Whenever changes take place in the structure and participants of AHP, the evaluation process has to be re-performed.

At times, decision-makers' true preferences might not have been reflected in the AHP process based on the methodology and the measuring scale used.

\section{Conclusion}

Although the ranking provided helps better understand the more significant criteria for CG implementation in Iran in the different domains, these criteria cannot really be separated from each other. For example, the two criteria of performance evaluation and training and development are directly related to each other. Based on the studies conducted on the subject, the process of identifying the training and development needs of personnel in organizations is associated with the performance evaluation process and its results, which is in fact the aim of developing personal development plans $(25,43)$. Furthermore, the three criteria of clinical effectiveness, clinical audit and risk management are also directly related to one another in a way that CG is the main component of the framework for risk management, control and governance in healthcare organizations, which can be achieved through the continuous promotion of clinical care standards, evidencebased clinical decision-making and reducing disparity of clinical results through clinical audits (17).

In addition, it is necessary to scrutinize the collective governance topic in CG implementation for factors such as resource allocation and personnel motivation. Involving other health departments such as the Food and Drug Organization in CG topics is an essential part of a better CG implementation. The researchers believe that a comprehensive, effective implementation of CG in the Iranian healthcare system requires a systematic and integrated view. This view must be based on up-to-date scientific findings and should systematically take account of changes in each component of the care providing system and their consequences for other components. Training planning and resource allocation must be performed more coherently based on the prioritized criteria of CG implantation and efforts should be made for improving training and creating 
a unique CG culture. The main requirements of CG implementation include having an effective policy at national levels, avoiding perfectionism, using the expertise and potentials of the entire country, emphasizing the prioritized criteria, and coordinating this model with other models of quality improvement such as accreditation and patient safety.

Ethical Issues

This work was supported by Tehran University of Medical sciences, Tehran, Iran [TUMS/SHMIS-1390/621].

\section{Competing interests}

Authors declare that they have no competing interests.

\section{Authors' contributions}

Conception and design: EH and HE. Acquisition of data: EH. Analysis and interpretation of data: $\mathrm{EH}$ and $\mathrm{HE}$. Drafting of the manuscript: MM and AVN. Critical revision of the manuscript for important intellectual content: ST and HR. Statistical analysis: EH and HE. Obtaining funding: ST. Administrative, technical, or material support: MM and AVN. Supervision: ST and HR.

\section{Authors' affiliations}

${ }^{1}$ Health Sciences Research Center, Department of Health and Management, School of Health, Mashhad University of Medical Sciences, Mashhad, Iran. ${ }^{2}$ Hospital Management Research Center, Iran University of Medical Sciences, Tehran, Iran. ${ }^{3}$ School of Health Management and Information Sciences, Iran University of Medical Sciences, Tehran, Iran. ${ }^{4}$ Department of Medical Records and Health Information Technology, School of Paramedical Sciences, Mashhad University of Medical Sciences, Mashhad, Iran.

\section{Endnotes}

1. In statistics, Cronbach alpha is used as a (lowerbound) estimate of the reliability of a psychometric test. Cronbach alpha will generally increase as the intercorrelations among test items increase, and is thus known as an internal consistency estimate of reliability of test scores.

\section{References}

1. Balding C. From quality assurance to clinical governance. Aust Health Rev 2008; 32: 383-91. doi: 10.1071/ah080383

2. Brennan M, Flynn A. Differentiating clinical governance, clinical management and clinical practice. Clinical Governance: An International Journal 2013; 18: 114-31. doi: 10.1108/14777271311317909

3. Scally G, Donaldson L. Clinical governance and drive for quality improvement. BMJ 1998; 317: 61-5. doi: 10.1136/ bmj.317.7150.61

4. Swage T. Clinical Care Takes center Stage. Nurs Times 1998; 94: 40-1.

5. Ellis B, Johnson S. The care pathway: a tool to enhance clinical governance. Clin Perform Qual Health Care 1999; 7: 134-44. doi: $10.1108 / 14664109920306820$

6. Robertson I, Edwards S. Gough M. Clinical governance in pre hospital care. Journal of Royal Society Medicine 2001; 94: 3842.

7. Baker R. Reforming primary care in England-again. Scand J Prim Health Care 2000; 18:72-4. doi: 10.1080/028134300750018936

8. Chandy J, Gooffellow T, Vohrah A. Clinical governance in action: radiology. Hosp Med 2000; 67: 326-9. doi: 10.12968/ hosp.2000.61.5.1331

9. Masterton RG, Teare EL. Clinical governance and infection control in the United Kingdom. J Hosp Infect 2001; 47: 25-31. doi: $10.1053 /$ jhin.2000.0858

10. Nicholls S, Cullen R, Oneill S. Clinical governance: it s origin and foundation. British Journal of Clinical Governance 2000; 5: 1728. doi: $10.1108 / 14777270010734055$
11. Webb V. One model of health care provision lessons learnt through clinical governance. J Forensic Leg Med 2010; 17: 36873. doi: $10.1016 / \mathrm{j} . j \mathrm{flm} .2010 .05 .011$

12. Hall $\mathrm{H}$, Curzio J. An investigation of change management process involved in the implementation of clinical governance by allied health professionals in Scotland. Physiother Res Int 2008;13: 205-6. doi: 10.1002/pri.425

13. Queensland Government. Clinical Governance Framework Key Policies and Implementation Standards. Queensland: Queensland Government; 2009. Available from: http://www. health.qld.gov.au

14. Patterson $\mathrm{L}$. The role of $\mathrm{CHI}$ in monitoring the development of clinical governance in trusts and promoting changes. British Journal of Clinical Governance 2002; 7: 147-9. doi: 10.1108/14664100210815477

15. MC sherry R, Pearce A, Tingle Y. Clinical Governance a Guide to Implementation For Health Care Professionals. London: John Wiley \& Sons; 2011.

16. Low Office of Ministry of Health and Medical Education. Tehran; 2009. Available from: http://lawoffice.mohme.gov.ir/lawoffice/ laws/dedicated_law/chapters_all.jsp?id=1

17. Patel S, Davies S. Identifying best practice principles of audit in health care. Nursing Standard 2010; 24: 40. doi: 10.7748/ ns2010.04.24.32.40.c7713

18. Grainger G, Hopkinson R, Barrett V. Implementing clinical governance- the result of a year's program of semi-structured visits to assess the development of clinical governance in West Midland Trusts. British Journal of Clinical Governance 2002; 7: 177-86. doi: 10.1108/14664100210438262

19. Specchia ML, La Torre G, Siliquini R, Capizzi S. OPTIGOV -Anew methodology for evaluating clinical governance implementation by health providers. BMC Health Serv Res 2010; 10: 174. doi: 10.1186/1472-6963-10-174

20. Department of Health. Clinical governance Guideline. Government of Western Australia; 2005.

21. Department of Health. Standards for better health. London; 2004. Available from http://www.nhserewash.com/safeguarding/ core\%20standards.pdf

22. Hooshmand E, Tourani S, Ravaghi H, Ebrahimipour $H$. Challenges in evaluating clinical governance systems in Iran: a qualitative study. Iran Red Crescent Med J 2014; 16: e13421. doi: $10.5812 /$ ircmj.13421

23. Ritter N. Understanding a widely misunderstood statistic: Cronbach's alpha. Paper presented at Southwestern Educational Research Association (SERA) Conference; New Orleans, LA; 2010.

24. Nunnally JC. Psychometric Theory. New York: McGraw-Hil; 1978.

25. Ibrahimipour $H$, Maleki $M$, Brown $R$, Gohari M, Karimi I, Dehnavieh R. A qualitative study of the difficulties in reaching sustainable universal health insurance coverage in Iran. Health Policy Plan 2011; 8: 1-11. doi: 10.1093/heapol/czq084

26. Ministray of Health and Medical Education (MoHME). State seminar of graduated specialists. Tehran; 2012.

27. Degeling P, Maxwell S, Macbeth F, Kennedy J. The impact of CHI: sense evidence From Wales. Qual Primary Care 2003; 11: 147-57.

28. Locock L. Redesigning health care: new wine from old bottles? J Health Serv Res Policy 2003; 8: 120-2. doi: $10.1258 / 135581903321466102$

29. Walshe K, Freeman T. Effectiveness of quality improvement: learning from evaluations. Qual safe Health care 2002; 11: 85-7. doi: 10.1136/qhc.11.1.85

30. Mohaghegh B, Ravaghi H, Mannion S. Clinical governance: implementation process and its challenges in Iranian hospitals. First International Congress on clinical governance and patient 
safety; Tehran, Iran; 2013.

31. Yosefan S, Najafi M, Kokabi F. Challenges of clinical goverment stablishment in health care organizations. First regional conference of clinical governance; Gorgan University of Medical Sciences; 2010.

32. Taylor L, Jones S. Clinical Governance in practice: closing the loop with integrated audit systems. Journal of Psychiatric and Medical Nursing 2006; 13: 228-33.

33. Khayatzadeh-Mahani A, Nekoei-Moghadam M, Esfandiari A, Ramezani F, Parva S. Clinical governance implementation: a developing country perspective. Clinical Governance: An International Journal 2013; 18(3): 186-99. doi: 10.1108/cgij-112012-0042

34. Friele R, Sluijs EM, Legemaate J. Complaint handeling in hospitals: an empirial stuy of discrepancies between patients expectations and their experiences. BMC Health Serv Res 2008; 8: 199. doi: 10.1186/1472-6963-8-199

35. Degeling P, Maxwell S, ledema R. Making clinical governance work. BMJ 2004; 1329: 679-81. doi: 10.1136/bmj.329.7467.679

36. Bendall-Lyon D, Powers TL. The role of complaint management in the service recovery process. Jt Comm J Qual Improv 2001; 27: 278-86.

37. Pichert JW, Miller CS, Hollo AH, Gauld-Jaeger J, Federspiel CF,
Hickson GB. What health professionals can do to identify and resolve patient dissatisfaction. Jt Comm J Qual Improv 1998; 24: 303-12.

38. Nayar P, Ozcan Y. Benchmarking urban acute care hospitals: efficiency and quality perspectives. Health Care Manag Rev 2013; 38: 137-45. doi: 10.1097/hmr.0b013e3182527a4c

39. Campbell M, Sheaff R, Sibbald B . Implementing clinical governance in english primary care groups: reconciling quality improvement and quality assurance. Qual Safe Health Care 2002; 11: 9-14. doi: 10.1136/qhc.11.1.9

40. Carl W. Principal to govern clinical governance. J Eval Clin Pract 2000; 6: 405-12. doi: 10.1046/j.1365-2753.2000.00248.x

41. Chandraharan E, Sabaratnam A. Clinical Governance. Obstetrics, Gynecology and Reproductive Medicine 2007; 17: 222-4. doi: 10.1016/j.ogrm.2007.05.003

42. Marshal M, Sheaff R, Rogers A. A qualitative study of the cultural changes in primary care organizations needed to implement clinical governance. British Journal of Medical Practice 2002; 52: $641-5$

43. Hard S, Schiller G, Aitken M. Continuous quality improvement: educating towards the culture of clinical governance. Qual Health Care 2001; 10: 70-8. 